\title{
Observador de Fluxos, Correntes e Ângulo de Carga de Máquinas Síncronas por meio da Filtragem de Partículas *
}

\author{
Iuri A. Monteiro* Letícia M. S. Vianna** \\ Mateus Giesbrecht ${ }^{* * *}$ \\ Faculdade de Engenharia Elétrica e de Computação, \\ Universidade Estadual de Campinas - UNICAMP \\ Campinas, SP \\ *monteiro@dsif.fee.unicamp.br \\ **leticiav@dsif.fee.unicamp.br \\ ***mateus@fee.unicamp.br
}

\begin{abstract}
The problem of the estimation of states of synchronous machines is directly related to the stability of electrical systems. Since the machines are highly non-linear systems, different methods of state observation seek to solve it in a timely manner and with satisfactory accuracy. This work presents a non-linear and recursive approach for the estimation of flux linkages, damping winding currents, and load angle of salient pole synchronous machines by means of particle filtering. A sixth order non-linear model is considered and only the measurements taken at the armature terminals during sinusoidal steady state are necessary to estimate the aformentioned states. To validate the method, the operation of a synchronous machine was simulated and the state estimation results obtained from the application of the algorithm validate the effectiveness of the proposed method.

Resumo: O problema da estimação de estados de máquinas síncronas se relaciona diretamente à estabilidade de sistemas elétricos. Por tratar-se de um sistema altamente não linear, diferentes métodos de observação de estados são utilizados para se determinarem seus estados em tempo hábil e com precisão satisfatória. Este trabalho apresenta uma abordagem não linear e aplicável recursivamente para a estimação de fluxos concatenados, correntes de enrolamentos amortecedores e ângulo de carga de máquinas síncronas de polos salientes por meio da filtragem de partículas. Um modelo não linear de sexta ordem é considerado e apenas as medições realizadas nos terminais da armadura durante regime permanente se fazem necessárias para estimar os referidos estados. Para validar o método, a operação de uma máquina síncrona foi simulada e os resultados de estimação de estado obtidos a partir da aplicação do algoritmo validam a eficácia do método proposto.
\end{abstract}

Keywords: Systems identification; electric systems; dynamic state estimation; particle filter; synchronous machines; on-line measurements; damper windings.

Palavras-chaves: Identificação de sistemas; sistemas elétricos; estimação dinâmica de estados; filtro de partículas; máquinas síncronas; medições em tempo real; enrolamentos amortecedores.

\section{INTRODUÇÃO}

As máquinas síncronas correspondem ao principal meio de geração de energia elétrica em todo mundo e, por este motivo, são consideradas um dos principais elementos integrantes de sistemas elétricos de potência (Glover et al., 2011). Deste modo, essas máquinas são capazes de influenciar consideravelmente o comportamento dos sistemas durante condições transitórias e de regime permanente.

Uma série de fatores pode levar os sistemas de potência a uma operação nos limiares técnicos de estabilidade, au-

* O presente trabalho foi realizado com apoio da Coordenação de Aperfeiçoamento de Pessoal de Nível Superior - Brasil (CAPES) Código de Financiamento 001 e do Conselho Nacional de Desenvolvimento Cientifico e Tecnológico - Brasil (CNPq). mentando a probabilidade de interrupções de serviço (Andersson et al., 2005). Grande parte desses eventos está associada a variações no ângulo de carga e à consequente dificuldade em manter-se o sincronismo dos geradores síncronos interligados aos referidos sistemas (Amin, 2001).

Para que o sincronismo seja mantido, a velocidade do rotor dos geradores síncronos precisam ser reguladas durante contingências, sem que se violem as condições de carga permissíveis (Hosseini et al., 2018). Os estabilizadores adotados em sistemas de potência fazem uso dos sinais de ângulo de carga para amortecer oscilações na potência de saída do gerador (Sumina et al., 2010). Assim, o conhecimento da velocidade do rotor, bem como do ângulo de carga, é essencial para a condução de estudos de estabilidade transitória (Kundur, 1994). 
Diferentes estimadores dinâmicos de estados vêm sendo propostos nas últimas décadas para a estimação de ângulos de carga e de outras grandezas das máquinas síncronas que influenciam na estabilidade do sistema, os quais se baseiam em diferentes implementações do Filtro de Kalman: o Filtro de Kalman Estendido (Ghahremani and Kamwa, 2016) e (Zhao et al., 2017); o Filtro de Kalman Unscented (Wang et al., 2012) e (Valverde et al., 2011). Sob certas condições - como conhecer a média, a covariância e a correlação dos ruídos de processo e de observação a cada instante de tempo -, esses filtros funcionam muito bem (Candy, 2009). Abordagens mais robustas como o Filtro de Kalman Estendido $\mathcal{H}_{\infty}$ (Zhao, 2018), capazes de tratar incertezas do sistema, também têm sido propostas.

Outro aspecto das máquinas síncronas que deve ser considerado é a corrente que flui nos enrolamentos amortecedores - barras de metal curto-circuitadas posicionadas em ranhuras nas faces polares. A medição dessas correntes é feita por meio de sensores que, se não estiverem previamente instalados, requerem que a máquina seja posta fora de operação para então instalá-los (Cuevas et al., 2018). Sendo assim, faz-se necessária a estimação dinâmica dessas correntes de modo a serem utilizadas na estimação de parâmetros de máquinas síncronas (Valverde et al., 2011).

O presente trabalho propõe a utilização do Filtro de Partículas, que é baseado no método de Monte Carlo, (Metropolis and Ulam, 1949) para a estimação do ângulo de carga e das correntes dos enrolamentos amortecedores de máquinas síncronas. O algoritmo é aplicado a dados obtidos por meio da simulação de um gerador síncrono de polos salientes em que se utilizam medições de tensões e correntes dos enrolamentos do estator e do rotor. Os resultados são verificados por meio da comparação entre os estados simulados e os estados estimados com o observador empregado.

O restante deste trabalho é estruturado da seguinte forma: na Seção 2 é apresentada uma breve discussão sobre um modelo em espaço de estado para a máquina síncrona de polos salientes. Na Seção 3 é apresentada a base teórica do filtro de partículas. Os resultados são apresentados e discutidos na Seção 4. Finalmente, as conclusões são resumidas na Seção 5 .

\section{MODELAGEM DE GERADORES SÍNCRONOS DE POLOS SALIENTES}

Os geradores síncronos são comumente construídos com enrolamentos trifásicos de armadura no estator e um enrolamento de excitação no rotor. Além desses enrolamentos, em máquinas de polos salientes, são construídas gaiolas de esquilo formadas por barras de cobre curto-circuitadas nas extremidades dos polos e eletricamente conectadas umas às outras. Esse enrolamento tem como função o amortecimento de transitórios da máquina e por esse motivo é conhecido como enrolamento amortecedor. Embora um número arbitrário de circuitos paralelos possa ser considerado para modelar a dinâmica do enrolamento amortecedor (Adkins, 1957), Krause et al. (2013) afirmam que o comportamento de máquinas síncronas de polos salientes é suficientemente predito por meio de um modelo com uma resistência e uma indutância equivalente em cada eixo.
As expressões desenvolvidas para as tensões e as correntes de máquinas síncronas formam um conjunto de equações diferenciais, cuja maioria dos coeficientes são funções periódicas do ângulo do rotor $\theta_{r}$ (Kundur, 1994). Mesmo nos casos em que se assume rotação constante, essas equações são trabalhosas e de difícil manipulação. Portanto, fazse uso de uma simples transformação de variáveis - a qual consiste na mudança do referencial $a b c$ do estator, para o referencial $q d 0$ fixo no rotor - para eliminar todas essas funções angulares das equações (Park, 1929). Por fim, partindo-se do pressuposto que o sistema em análise opera em condições balanceadas de carga, as componentes de sequência zero podem ser consideradas iguais a zero (Adkins, 1957) e, portanto, não serão apresentadas neste trabalho.

A referida transformação matricial é:

$$
\mathbf{f}_{q d}=\mathbf{K} \mathbf{f}_{a b c}
$$

em que $\mathbf{f}_{q d}=\left[\begin{array}{ll}f_{q} & f_{d}\end{array}\right]^{\mathrm{T}}, \mathbf{f}_{a b c}=\left[\begin{array}{lll}f_{a} & f_{b} & f_{c}\end{array}\right]^{\mathrm{T}}$,

$$
\mathbf{K}=\frac{2}{3}\left[\begin{array}{ccc}
\cos \theta_{r} & \cos \left(\theta_{r}-2 \pi / 3\right) & \cos \left(\theta_{r}-4 \pi / 3\right) \\
\operatorname{sen} \theta_{r} & \operatorname{sen}\left(\theta_{r}-2 \pi / 3\right) & \operatorname{sen}\left(\theta_{r}-4 \pi / 3\right)
\end{array}\right],
$$

e o ângulo $\theta_{r}$, em radianos, é dado por

$$
\theta_{r}=\omega_{s} t+\delta-\pi / 2 .
$$

Nas equações acima, $f$ pode representar tanto tensões, correntes e fluxos concatenados, quanto cargas elétricas. $\mathrm{O}$ sobrescrito $\mathrm{T}$ denota a transposta de uma matriz. Além disso, $\omega_{s}$ é a velocidade angular síncrona, em radianos elétricos por segundo, e $\delta$, o ângulo de carga (deslocamento angular do rotor entre o eixo em quadratura e o campo girante da armadura (IEEE-100, 2000)), em radianos.

As equações de tensão no referencial $q d$ são

$$
\begin{aligned}
\mathbf{v}_{q d s} & =\mathbf{r}_{s} \mathbf{i}_{q d s}+\frac{\mathrm{d}}{\mathrm{d} t} \boldsymbol{\psi}_{q d s}+\omega_{r} \boldsymbol{\psi}_{d q s} \\
\mathbf{v}_{q d r} & =\mathbf{r}_{r} \mathbf{i}_{q d r}+\frac{\mathrm{d}}{\mathrm{d} t} \boldsymbol{\psi}_{q d r} .
\end{aligned}
$$

A Equação (4) se refere às tensões nos enrolamentos do estator, nas quais $\mathbf{v}_{q d s}=\left[\begin{array}{ll}v_{q} & v_{d}\end{array}\right]^{\mathrm{T}}, \mathbf{i}_{q d s}=\left[\begin{array}{ll}i_{q} & i_{d}\end{array}\right]^{\mathrm{T}}$, $\boldsymbol{\psi}_{q d s}=\left[\psi_{q} \psi_{d}\right]^{\mathrm{T}}, \boldsymbol{\psi}_{d q s}=\left[\psi_{d} \psi_{q}\right]^{\mathrm{T}}, \mathrm{e} \mathbf{r}_{s}=\operatorname{diag}\left[r_{s} r_{s}\right]$. Por outro lado, (5) representa as equações do rotor, em que $\mathbf{v}_{q d r}=\left[\begin{array}{lll}v_{k q} & v_{f d} & v_{k d}\end{array}\right]^{\mathrm{T}}, \mathbf{i}_{q d r}=\left[\begin{array}{lll}i_{k q} & i_{f d} & i_{k d}\end{array}\right]^{\mathrm{T}}, \boldsymbol{\psi}_{q d r}=$ $\left[\psi_{k q} \psi_{f d} \psi_{k d}\right]^{\mathrm{T}}$, e $\mathbf{r}_{r}=\operatorname{diag}\left[r_{k q} r_{f d} r_{k d}\right]$. Além disso, $v, i, \psi, r$ se referem a tensões, correntes, fluxos concatenados e resistências, respectivamente. Os índices $s, k q, f d, k d$ indicam onde cada uma das grandezas é observada: enrolamentos da armadura, amortecedor de eixo em quadratura, de campo e amortecedor de eixo direto, respectivamente.

Em (4) e (5), $\omega_{r}$ é a velocidade do rotor e, assim como todas as outras grandezas, estão em por unidade (p.u.).

Em termos práticos, é conveniente expressar as tensões $v$ e fluxos concatenados $\psi$ em termos de reatâncias $x$ e fluxos concatenados por segundo $\Psi$, os quais são definidos como

$$
\begin{aligned}
\Psi_{q} & =x_{l s} i_{q}+x_{m q}\left(i_{q}+i_{k q}\right), \\
\Psi_{d} & =x_{l s} i_{q}+x_{m d}\left(i_{d}+i_{f d}+i_{k d}\right), \\
\Psi_{k q} & =x_{l k q} i_{k q}+x_{m q}\left(i_{q}+i_{k q}\right), \\
\Psi_{f d} & =x_{l f d} i_{f d}+x_{m d}\left(i_{d}+i_{f d}+i_{k d}\right), \\
\Psi_{k d} & =x_{l k d} i_{k d}+x_{m d}\left(i_{d}+i_{f d}+i_{k d}\right) .
\end{aligned}
$$


Em (6)-(10), as reatâncias que apresentam o subscrito $l$ representam reatâncias de dispersão; o subscrito $m$, por sua vez, refere-se à componente de magnetização.

As definições (11) e (12) de fluxos concatenados de magnetização por segundo de eixo em quadratura e eixo direto são, respectivamente,

$$
\begin{aligned}
& \Psi_{m q}=x_{a q}\left(\frac{\Psi_{q}}{x_{l s}}+\frac{\Psi_{k q}}{x_{l k q}}\right) \\
& \Psi_{m d}=x_{a d}\left(\frac{\Psi_{d}}{x_{l s}}+\frac{\Psi_{f d}}{x_{l f d}}+\frac{\Psi_{k d}}{x_{l k d}}\right),
\end{aligned}
$$

em que

$$
\begin{aligned}
& x_{a q}=\left(\frac{1}{x_{m q}}+\frac{1}{x_{l s}}+\frac{1}{x_{l k q}}\right)^{-1}, \\
& x_{a d}=\left(\frac{1}{x_{m d}}+\frac{1}{x_{l s}}+\frac{1}{x_{l f d}}+\frac{1}{x_{l k d}}\right)^{-1},
\end{aligned}
$$

permitem que as correntes nos enrolamentos sejam expressas em termos dos fluxos de dispersão dos enrolamentos e de magnetização como

$$
\begin{aligned}
i_{q} & =\frac{1}{x_{l s}}\left(\Psi_{q}-\Psi_{m q}\right), \\
i_{d} & =\frac{1}{x_{l s}}\left(\Psi_{d}-\Psi_{m d}\right), \\
i_{k q} & =\frac{1}{x_{k q}}\left(\Psi_{k q}-\Psi_{m q}\right), \\
i_{f d} & =\frac{1}{x_{f d}}\left(\Psi_{f d}-\Psi_{m d}\right), \\
i_{k d} & =\frac{1}{x_{k d}}\left(\Psi_{k d}-\Psi_{m d}\right) .
\end{aligned}
$$

Resolvendo (4) e (5) para os fluxos concatenados por segundo e considerando (15)-(19), podem-se escrever as equações de estado para os enrolamentos do estator e do rotor:

$$
\begin{aligned}
\dot{\Psi}_{q} & =\omega_{b}\left[v_{q}-\frac{\omega_{r}}{\omega_{b}} \Psi_{d}+\frac{r_{s}}{x_{l s}}\left(\Psi_{m q}-\Psi_{q}\right)\right], \\
\dot{\Psi}_{d} & =\omega_{b}\left[v_{d}+\frac{\omega_{r}}{\omega_{b}} \Psi_{q}+\frac{r_{s}}{x_{l s}}\left(\Psi_{m d}-\Psi_{d}\right)\right], \\
\dot{\Psi}_{k q} & =\omega_{b}\left[v_{k q}+\frac{r_{k q}}{x_{l k q}}\left(\Psi_{m q}-\Psi_{k q}\right)\right], \\
\dot{\Psi}_{f d} & =\omega_{b}\left[v_{f d}+\frac{r_{f d}}{x_{l f d}}\left(\Psi_{m d}-\Psi_{f d}\right)\right], \\
\dot{\Psi}_{k d} & =\omega_{b}\left[v_{k d}+\frac{r_{k d}}{x_{l k d}}\left(\Psi_{m d}-\Psi_{k d}\right)\right],
\end{aligned}
$$

em que $\omega_{b}$ é a velocidade angular base utilizada para se calcularem as reatâncias indutivas, em radianos por segundo.

Devido à dificuldade de se medir o ângulo de carga, sua dinâmica também é considerada e este ângulo é adicionado ao vetor de estados como um parâmetro a ser determinado. De (3),

$$
\dot{\delta}=\omega_{b} \omega_{r}-\omega_{s},
$$

em que $\omega_{b}$ foi adicionado a (3) para se considerar $\dot{\delta}$ em radianos por segundo.

\subsection{Representação em Espaço de Estado}

Uma formulação genérica e determinística de um sistema dinâmico, multivariável e não linear, incluindo o modelo das observações em espaço de estado, pode ser dada por

$$
\begin{aligned}
& \dot{\mathbf{x}}=f(\mathbf{x}, \mathbf{u}), \\
& \mathbf{y}=h(\mathbf{x}),
\end{aligned}
$$

em que $\mathbf{x}, \mathbf{y}$, e u são os vetores de estados (de dimensão $n$ ), observações (de dimensão $l$ ) e entradas (de dimensão $m$ ) com as correspondentes funções de processo, observação e entrada (Candy, 2009).

Para máquinas síncronas, o modelo mais amplamente utilizado é derivado das equações de tensões expressas no referencial $q d$ com os fluxos concatenados por segundo como variáveis de estado. Este modelo pode ser encontrado em detalhes em (Krause et al., 2013). Consideram-se, portanto, os seguintes vetores de estados, observações e entradas

$$
\begin{aligned}
& \mathbf{x}=\left[\begin{array}{lllll}
\Psi_{q} & \Psi_{d} & \Psi_{k q} & \Psi_{f d} & \Psi_{k d} \delta
\end{array}\right]^{\mathrm{T}}, \\
& \mathbf{y}=\left[\begin{array}{lllll}
i_{q} & i_{d} & i_{k q} & i_{f d} & i_{k d}
\end{array}\right]^{\mathrm{T}}, \\
& \mathbf{u}=\left[\begin{array}{llllll}
v_{q} & v_{d} & v_{k q} & v_{f d} & v_{k d} & \omega_{r}
\end{array}\right]^{\mathrm{T}} .
\end{aligned}
$$

Haja vista que amostrar sistemas contínuos é uma prática comum, o sistema amostrado em espaço de estados se torna

$$
\begin{aligned}
\mathbf{x}_{k+1} & =f\left(\mathbf{x}_{k}, \mathbf{u}_{k}\right), \\
\mathbf{y}_{k} & =h\left(\mathbf{x}_{k}\right),
\end{aligned}
$$

de modo que o subscrito $k$ indica valores no instante de tempo $t_{k}=k T$, em que $T$ o valor inverso da frequência de amostragem. Ressalta-se que neste trabalho, utilizase a aproximação de série de Taylor de primeira ordem para a determinação da função de transição de estado discreta (31).

\section{O FILTRO DE PARTÍCULAS}

O Filtro de Partículas pertence a uma classe de filtros estocásticos, sendo uma técnica de Monte Carlo para a solução do problema de estimação de estados e parâmetros (Candy, 2009). O filtro baseia-se no estimador Bayesiano, que trata de problemas não lineares e não gaussianos.

A primeira manifestação científica do Filtro de Partículas data de Gordon et al. (1993), ora denominado bootstrap filter e, atualmente, Sampling Importance Resampling (Salmond and Gordon, 2005). A filtragem de partículas pode ser dividida em duas etapas: propagação e atualização (Gordon et al., 1993; Gustafsson, 2010). Essa abordagem se utiliza da técnica de amostragem por importância na seleção das partículas mais representativas que irão, posteriormente, gerar as novas amostras da função de densidade de probabilidade desejada (pdf, do inglês probability density function) (Candy, 2009).

Seja o sistema

$$
\begin{aligned}
\mathbf{x}_{k+1} & =f_{k}\left(\mathbf{x}_{k}, \boldsymbol{\eta}_{k}\right), \\
\mathbf{y}_{k} & =h_{k}\left(\mathbf{x}_{k}, \boldsymbol{\nu}_{k}\right),
\end{aligned}
$$

em que $k$ é o instante de tempo; $\boldsymbol{\eta}_{k}$ é o vetor de ruído de processo; e $\boldsymbol{\nu}_{k}$, o vetor de ruído de observação. Supõemse que os ruídos são independentes e brancos e possuem pdf conhecidas. As funções $f_{k}(\cdot)$ e $h_{k}(\cdot)$ são variantes 
no tempo, não lineares e representam o sistema e as observações, respectivamente.

O objetivo do estimador Bayesiano é aproximar a pdf condicional $p\left(\mathbf{x}_{k} \mid Y_{k}\right)$, em que $\mathbf{x}_{k}$ baseia-se nas medições $Y_{k}=\left\{\mathbf{y}_{1}, \mathbf{y}_{2}, \ldots, \mathbf{y}_{k}\right\}$ e $Y_{k}$ é o espaço que contém todas as observações até o instante de tempo $k$.

Para a inicialização do problema de estimação, gera-se uma quantidade $N$ de vetores de estados baseados na pdf inicial $p\left(\mathbf{x}_{0}\right)$, que supõe-se conhecida. Este vetor de estados recebe o nome de partícula, sendo definido pelo projetista e denotado por $\mathbf{x}_{0, i}^{+}, i=1, \ldots, N$. Como a primeira medição é encontrada no instante de tempo $k=0$, a pdf do instante inicial é $p\left(\mathbf{x}_{0}\right)=p\left(\mathbf{x}_{0} \mid Y_{0}\right)$.

Para cada instante de tempo $k=1,2, \ldots$, realiza-se a propagação das partículas para o próximo instante por meio de

$$
\mathbf{x}_{k, i}^{-}=f_{k-1}\left(\mathbf{x}_{k-1, i}^{+}, \boldsymbol{\eta}_{k-1, i}\right),
$$

em que o ruído de processo $\eta_{k-1, i}$ é gerado aleatoriamente com base na pdf de $\boldsymbol{\eta}_{k-1}$; e $\mathbf{x}_{k, i}^{-}$são as partículas a priori. Posteriormente, calculam-se a observação para o instante $k, p\left(\mathbf{y}_{k} \mid \mathbf{x}_{k, i}^{-}\right)$, e os pesos $w_{i}$ para cada uma das partículas. O peso para a partícula $i$ é dado por

$$
w_{i}=\frac{1}{(2 \pi)^{l / 2}|\mathbf{R}|^{0.5}} \exp \left[\frac{-\left(y_{k}^{*}-\bar{y}_{k}\right)^{T} \mathbf{R}^{-1}\left(y_{k}^{*}-\bar{y}_{k}\right)}{2}\right],
$$

em que $y_{k}^{*}$ é a observação real; $\bar{y}_{k}=h\left(\mathbf{x}_{k, i}^{-}\right)$é a observação estimada; e $\mathbf{R}$ é a covariância do ruído de observação, $\boldsymbol{\eta}_{k} \sim \mathcal{N}(0, \mathbf{R})$. É importante ressaltar que o peso $w_{i}$ é uma medida direta de verossimilhança (Simon, 2006).

Em seguida, os pesos são normalizados conforme

$$
w_{i}=\frac{w_{i}}{\sum_{j=1}^{N} w_{j}} .
$$

A seleção das partículas mais significativas se dá por um processo de reamostragem que considera a função de densidade acumulada e gera o conjunto de partículas a posteriori, $\mathbf{x}_{k, i}^{+}$. Desse modo, garante-se que este novo conjunto de partículas está distribuído conforme a pdf $p\left(\mathbf{x}_{k} \mid Y_{k}\right)$. A partir deste conjunto de partículas a posteriori, calculase alguma medida estatística para estimar os estados comumente, utiliza-se a média.

\subsection{Aplicação do Filtro de Partículas à Estimação de Estados da Máquina Síncrona de Polos Salientes}

Uma vez que o Filtro de Partículas se baseia no cálculo de pesos e, portanto, na análise da verossimilhança entre os valores observados e os valores medidos, faz-se necessária a inclusão de valores medidos do ângulo de carga no vetor de observações, como em (Valverde et al., 2011). Como este trabalho utiliza resultados de simulação para validarem o desempenho do Filtro de Partículas, medidas do ângulo de carga se encontram à disposição para a supracitada inclusão. Se uma máquina síncrona real fosse utilizada, faria-se necessária a utilização de técnicas, tais como a desenvolvida em (Kim et al., 2018), para se medir o ângulo de carga.

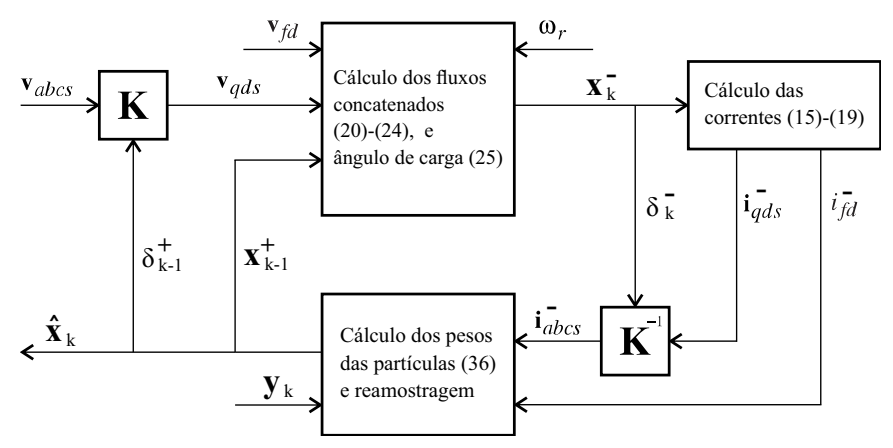

Figura 1. Fluxograma simplificado do procedimento de filtragem de partículas aplicado à estimação de estados da máquina síncrona.

Além disso, as tensões $v_{k q}$ e $v_{k d}$ de (30) são ajustadas a zero, uma vez que representam quedas de tensão em enrolamentos curto-circuitados. Portanto, os vetores de estado, observação e entrada se tornam, respectivamente:

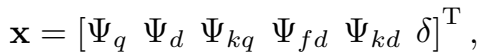

$$
\begin{aligned}
& \mathbf{y}=\left[\begin{array}{lllll}
i_{a} & i_{b} & i_{c} & i_{f d} & \delta_{m}
\end{array}\right]^{\mathrm{T}} \text {, } \\
& \mathbf{u}=\left[\begin{array}{lllllll}
v_{a} & v_{b} & v_{c} & 0 & v_{f d} & 0 & \omega_{r}
\end{array}\right]^{\mathrm{T}},
\end{aligned}
$$

em que $\delta_{m}$ é o valor medido do ângulo de carga.

O fluxograma da Figura 1 resume o procedimento da filtragem de partículas aplicado à estimação de estados da máquina síncrona de polos salientes.

\section{RESULTADOS DA APLICAÇÃO DO FILTRO DE PARTÍCULAS}

Um modelo de um gerador síncrono de polos salientes de 126 MVA, 8 pares polares e $13,8 \mathrm{kV}$ foi utilizado para validar o desempenho da abordagem via Filtro de Partículas proposta. O gerador síncrono foi simulado por meio do Simulink® em paralelo com o barramento infinito. Os dados da simulação foram somados a ruídos brancos de média nula e variância 0,001 para simular os ruídos que seriam observados em uma aplicação real. As simulações foram executadas em um computador de processador Intel@ Core $^{\mathrm{TM}}$ i7-4500Ux64 CPU @ 1,80 GHz, 8,00 GB de memória RAM, executando o sistema operacional Microsoft(C) Windows 10.

Os parâmetros utilizados na simulação encontram-se disponíveis na folha de dados do fornecedor e são apresentados na Tabela 1 . As medições foram coletadas durante $0,2 \mathrm{~s}$ em condições de regime permanente, saturação e ângulo de carga $\delta=0,525 \mathrm{rad}$, com frequência de amostragem igual a $10 \mathrm{kHz}$ - resultando em 2000 amostras. O conjunto de medições inclui as tensões e correntes em corrente alternada nos terminais da armadura; e a tensão e a corrente em corrente contínua injetada no enrolamento de campo.

A seleção da matriz de covariância $\mathbf{R}$ depende do nível de incerteza das medições. A fim de se utilizar um ruído de observação independente e identicamente distribuído, os elementos da diagonal principal da matriz $\mathbf{R}$ foram mantidos constantes e iguais a 0,001. O estado inicial $\mathbf{x}_{0}$, por sua vez, foi gerado aleatoriamente a partir de uma função de distribuição gaussiana de média nula e variância igual a 0,01 . 

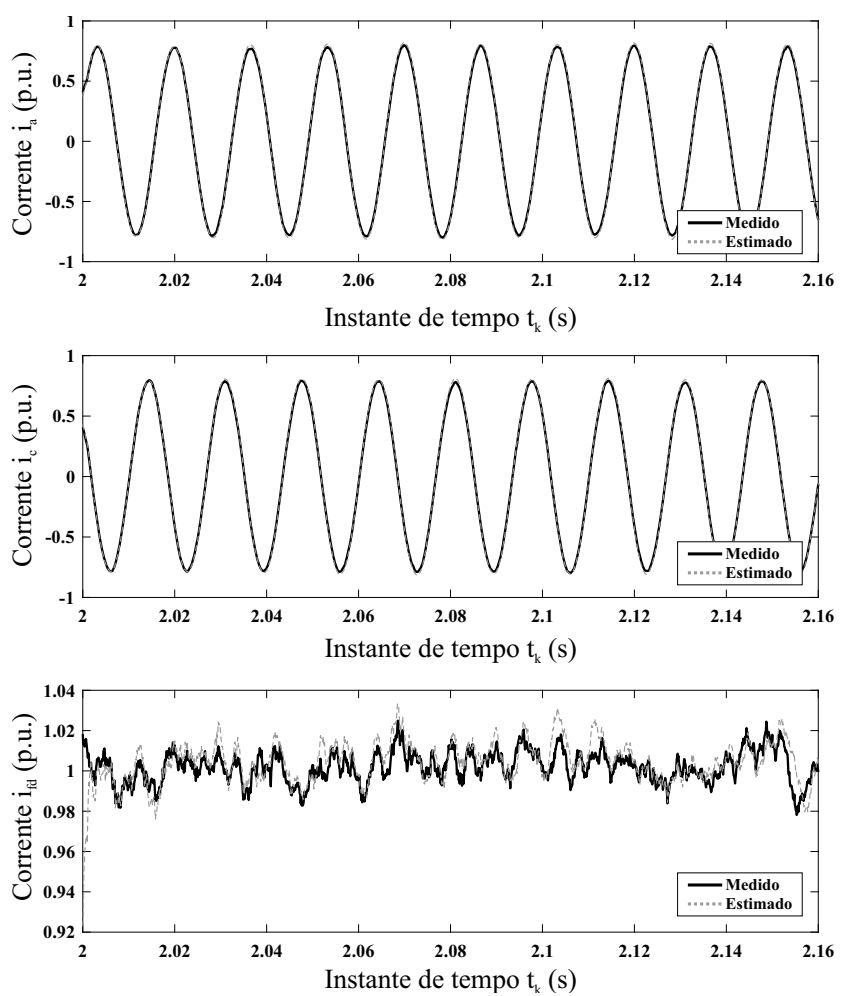
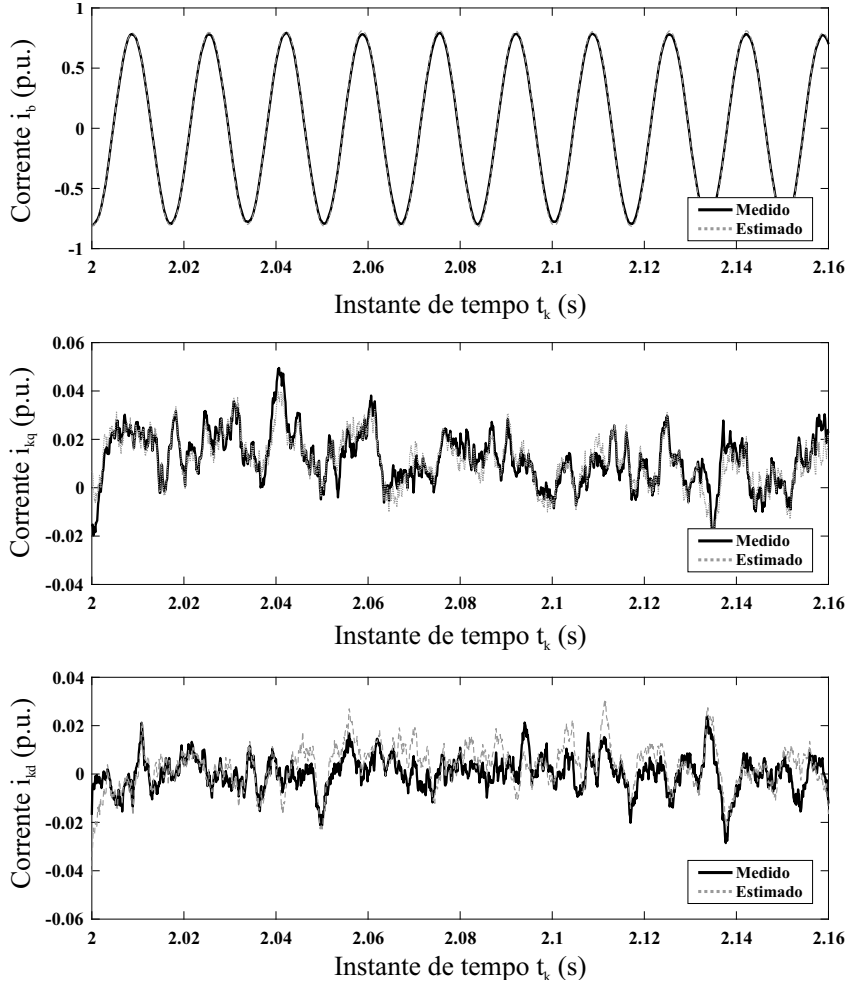

Figura 2. Estimação das correntes dos enrolamentos da máquina síncrona em condições de regime permanente. As curvas contínuas, em preto, representam os valores medidos e as curvas pontilhadas, em cinza, os valores estimados. A primeira coluna apresenta as correntes $i_{a}, i_{c}$ e $i_{f d}$, enquanto a segunda coluna apresenta as correntes $i_{b}, i_{k q}$ e $i_{k d}$.

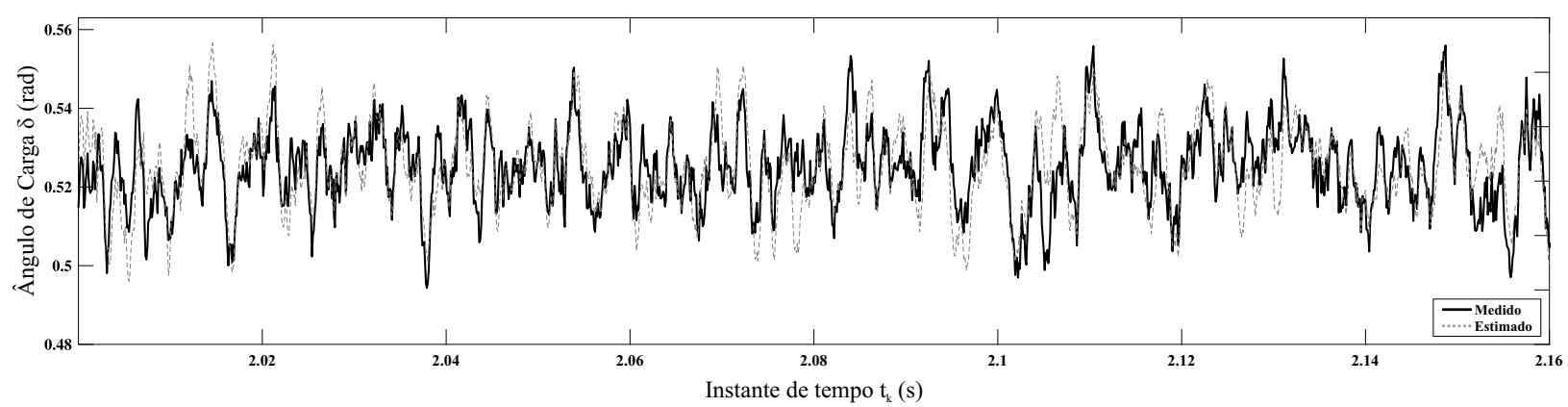

Figura 3. Estimação do ângulo de carga $\delta$. A curva contínua, em preto, representa os valores medidos e a curva pontilhada, em cinza, os valores estimados.

Tabela 1. Parâmetros do gerador síncrono simulado conforme a folha de dados do fornecedor.

\begin{tabular}{cccc}
\hline Parâmetro & Valor (p.u.) & Parâmetro & Valor (p.u.) \\
\hline$r_{s}$ & 0,00120 & $x_{l k q}$ & 0,1645 \\
$r_{k q}$ & 0,02993 & $x_{l f d}$ & 0,1507 \\
$r_{f d}$ & 0,00027 & $x_{l k d}$ & 0,1097 \\
$r_{k d}$ & 0,01995 & $x_{m q}$ & 0,5146 \\
$x_{l s}$ & 0,1180 & $x_{m d}$ & 0,9530 \\
\hline
\end{tabular}

Diferentes quantidades de partículas foram usadas para avaliar a relação entre esforço computacional e eficiência da estimação. Na Tabela 2, resumem-se os resultados encontrados para erros quadráticos médios das estimações das correntes dos enrolamentos amortecedores $i_{k q}$ e $i_{k d}$ e do ângulo de carga $\delta$, bem como o tempo de execução do algoritmo de estimação. É possível perceber que quanto
Tabela 2. Erro quadrático médio e tempo de execução para diferentes quantidades de partículas.

\begin{tabular}{ccccc}
\hline $\mathrm{N}$ & $i_{k q}$ & $i_{k d}$ & $\delta$ & Tempo (s) \\
\hline 100 & 0,4548 & 0,5221 & 0,4675 & 19 \\
500 & 0,2640 & 0,3135 & 0,2754 & 93 \\
1000 & 0,2209 & 0,2675 & 0,2435 & 189 \\
5000 & 0,1578 & 0,1927 & 0,2121 & 1011 \\
\hline
\end{tabular}

maior o número de partículas, maior o tempo de execução do algoritmo, mas menor o erro quadrático médio.

Ressalta-se também que, para o computador utilizado nas simulações, não seria possível fazer uma análise em tempo real. Entretanto, haja vista a eficiência do método proposto, o algoritmo pode ser invocado intermitentemente para que estimavas em tempo real sejam realizadas. 
Na Figura 2, apresentam-se as observações estimadas (elementos do vetor $\mathbf{y}$, definido em (39)) e os respectivos valores medidos. É importante destacar a presença dos ruídos de observação e de processo adicionados aos sinais medidos, conforme (33) e (34) e conforme seria observado em um caso real. Das figuras, notam-se que as estimativas apresentam comportamento similar aos valores observados. Na Figura 3, pode-se verificar a pequena variação existente entre os valores real e estimado para o ângulo de carga. A dimensão da variação não traz problemas de estabilidade, uma vez que não ultrapassa as margens técnicas de estabilidade (Kundur, 1994). Destaca-se, ainda, a amplitude dos valores medidos, variando entre 0,495 e 0,558 radianos.

\section{CONCLUSÕES}

Neste trabalho, uma abordagem para a estimação não linear de fluxos concatenados, correntes de enrolamentos amortecedores e ângulo de carga de máquinas síncronas de polos salientes por meio do Filtro de Partículas foi proposta. Verificou-se, por meio da aplicação do algoritmo proposto a dados obtidos da simulação da máquina síncrona acrescidos de ruído, que o procedimento é capaz de filtrar observações altamente ruidosas ao mesmo tempo em que estima os supracitados estados com precisão considerável. O método recursivo é simples de ser executado e não requer transformações ou linearizações para lidar com as equações não lineares e, portanto, pode ser utilizado para aplicações reais. O próximo passo da pesquisa será a aplicação dos algoritmo desenvolvido a dados obtidos em uma máquina síncrona real.

\section{REFERÊNCIAS}

Adkins, B. (1957). The General Theory of Electrical Machines. Associated Electrical Industries Series. Chapman \& Hall LTD, London.

Amin, M. (2001). Toward self-healing energy infrastructure systems. IEEE Computer Applications in Power, 14(1), 20-28. doi:10.1109/67.893351.

Andersson, G., Donalek, P., Farmer, R., Hatziargyriou, N., Kamwa, I., Kundur, P., Martins, N., Paserba, J., Pourbeik, P., Sanchez-Gasca, J., Schulz, R., Stankovic, A., Taylor, C., and Vittal, V. (2005). Causes of the 2003 major grid blackouts in north america and europe, and recommended means to improve system dynamic performance. IEEE Transactions on Power Systems, 20(4), 1922-1928. doi:10.1109/TPWRS.2005.857942.

Candy, J.V. (2009). Bayesian Signal Processing: Classical, Modern, and Particle Filtering Methods.

Cuevas, M., Romary, R., Lecointe, J., Morganti, F., and Jacq, T. (2018). Noninvasive detection of winding shortcircuit faults in salient pole synchronous machine with squirrel-cage damper. IEEE Transactions on Industry Applications, 54(6), 5988-5997. doi:10.1109/TIA.2018. 2861860.

Ghahremani, E. and Kamwa, I. (2016). Local and widearea pmu-based decentralized dynamic state estimation in multi-machine power systems. IEEE Transactions on Power Systems, 31(1), 547-562. doi:10.1109/TPWRS. 2015.2400633.

Glover, J., Sarma, M., and Overbye, T. (2011). Power System Analysis and Design. Cengage Learning.
Gordon, N.J., Salmond, D.J., and Smith, A.F. (1993). Novel approach to nonlinear/non-gaussian bayesian state estimation. In IEE proceedings $F$ (radar and signal processing), volume 140, 107-113. IET.

Gustafsson, F. (2010). Particle filter theory and practice with positioning applications. IEEE Aerospace and Electronic Systems Magazine, 25(7), 53-82.

Hosseini, S.M., Abdollahi, R., and Karrari, M. (2018). Inclusive design and implementation of online load angle measurement for real-time transient stability improvement of a synchronous generator in a smart grid. IEEE Transactions on Industrial Electronics, 65(11), 89668972. doi:10.1109/TIE.2018.2811394.

IEEE-100 (2000). The authoritative dictionary of ieee standards terms, seventh edition. IEEE Std 100-2000, 1-1362. doi:10.1109/IEEESTD.2000.322230.

Kim, D., Moon, Y., Lee, J., Ryu, H., and Kim, T. (2018). A new method of recording generator dynamics and its application to the derivation of synchronous machine parameters for power system stability studies. IEEE Transactions on Energy Conversion, 33(2), 605-616. doi:10.1109/TEC.2017.2772234.

Krause, P., Wasynczuk, O., Sudhoff, S., and Pekarek, S. (2013). Analysis of Electric Machinery and Drive Systems. IEEE Press Series on Power Engineering. John Wiley \& Sons, 3 edition.

Kundur, P. (1994). Power Systems Stability and Control. Power System Engineering Series. Electric Power Research Institute.

Metropolis, N. and Ulam, S. (1949). The monte carlo method. Journal of the American Statistical Association, 44(247), 335-341. doi:10.1080/01621459.1949. 10483310. PMID: 18139350.

Park, R.H. (1929). Two-reaction theory of synchronous machines generalized method of analysis-part i. Transactions of the American Institute of Electrical Engineers, 48(3), 716-727.

Salmond, D. and Gordon, N. (2005). An introduction to particle filters. State space and unobserved component models theory and applications, 1-19.

Simon, D. (2006). Optimal state estimation: Kalman, $\mathcal{H}_{\infty}$, and nonlinear approaches. John Wiley \& Sons.

Sumina, D., Bulić, N., and Erceg, I. (2010). Threedimensional power system stabilizer. Electric power systems research, 80(7), 886-892.

Valverde, G., Kyriakides, E., Heydt, G.T., and Terzija, V. (2011). Nonlinear estimation of synchronous machine parameters using operating data. IEEE Transactions on Energy Conversion, 26(3), 831-839. doi:10.1109/TEC. 2011.2141136.

Wang, S., Gao, W., and Meliopoulos, A.P.S. (2012). An alternative method for power system dynamic state estimation based on unscented transform. IEEE Transactions on Power Systems, 27(2), 942-950. doi:10.1109/ TPWRS.2011.2175255.

Zhao, J. (2018). Dynamic state estimation with model uncertainties using $h_{\infty}$ extended kalman filter. IEEE Transactions on Power Systems, 33(1), 1099-1100. doi: 10.1109/TPWRS.2017.2688131.

Zhao, J., Netto, M., and Mili, L. (2017). A robust iterated extended kalman filter for power system dynamic state estimation. IEEE Transactions on Power Systems, 32(4), 3205-3216. doi:10.1109/TPWRS.2016.2628344. 